\title{
Double Skin Facade Integrated Damping Systems for Tall Buildings
}

\author{
Kyoung Sun Moon \\ School of Architecture \\ Yale University, New Haven, CT, USA
}

\begin{abstract}
As buildings become ever taller and more slender, wind-induced vibration is a serious design issue. This paper studies integrated damping systems for tall buildings utilizing double skin facades (DSF), which are composed of two layers of facades with substantial gaps between them and whose uses have been increasing due to their superior environmental performance. Two schemes are studied. In the first scheme, the connectors between the inner and outer skins of the DSF system are designed to have low axial stiffness with a damping mechanism. Through this design, the vibration of the primary building structure can be substantially reduced. However, the excessive movements of the DSF outer skin masses are a critical design limitation. In the second scheme, instead of utilizing the movements of the DSF outer skin masses, small tuned mass dampers (TMDs) are installed within the DSF cavities. Compared with the conventional TMD systems, which are typically installed by occupying very valuable large space near the top of tall buildings, this scheme does not sacrifice any interior space for TMDs. However, effectiveness of the system is reduced to some degree as the multiple small TMDs are distributed vertically along the height of the building and the movements of the TMD masses are limited within the DSF cavities. Further research is required for practical application of the integrated damping systems studied in this paper.
\end{abstract}

Keywords - Tall Buildings, Double Skin Facades, Damping, Tuned Mass Dampers

\section{INTRODUCTION}

Double skin façade (DSF) systems, which have substantial cavity space between the inner and outer façade layers, have obtained increased interest due to their superior performance as one of the most advanced environmental mediators. Many tall buildings have been built with DSF systems including the ARAG 2000 Tower in Dusseldorf, Business Tower in Nuremberg, RWE Tower in Essen [1], and more recently completed Shanghai Tower in Shanghai. At its height of $632 \mathrm{~m}$, the Shanghai Tower is the tallest building with a DSF system in the world and also this building's DSF system has the widest gap between the inner and outer façade layers.

While many studies have been carried out regarding environmental performances of the DSF system, investigations on the structural capability of the DSF system are rare. In tall buildings, excessive wind-induced vibrations can cause serious human discomfort problem. This paper studies tall building dynamic motion control utilizing DSF systems by introducing energy dissipating mechanisms within their cavities.

Moon (2009) studied the potential of DSF systems as a damping mechanism for tall buildings [2]. This paper briefly reviews this study first. The DSF damping system is based on low axial stiffness connectors and damping mechanism between the inner and outer skins. Optimal tuning and design challenges of the DSF damping system are discussed.

Though the DSF damping system can be designed to significantly reduce the lateral vibration of tall buildings, excessive movements of the DSF outer skin masses due to the low axial stiffness connectors are a serious design issue. In order to mitigate this challenge, installing small tuned mass dampers (TMDs) within the DSF cavity spaces along the building height is studied. The TMD system, which effectively reduces vibrations of tall buildings, is usually composed of one or two large TMD masses located near the top for better performance. As a result, very valuable occupiable space near the top of tall buildings is sacrificed to contain large TMDs. By distributing multiple smaller TMDs within the DSF cavities, this conventional TMD related design issue is eliminated. Through design studies, effectiveness of the DSF integrated TMD system is investigated in comparison with the conventional TMD system. 


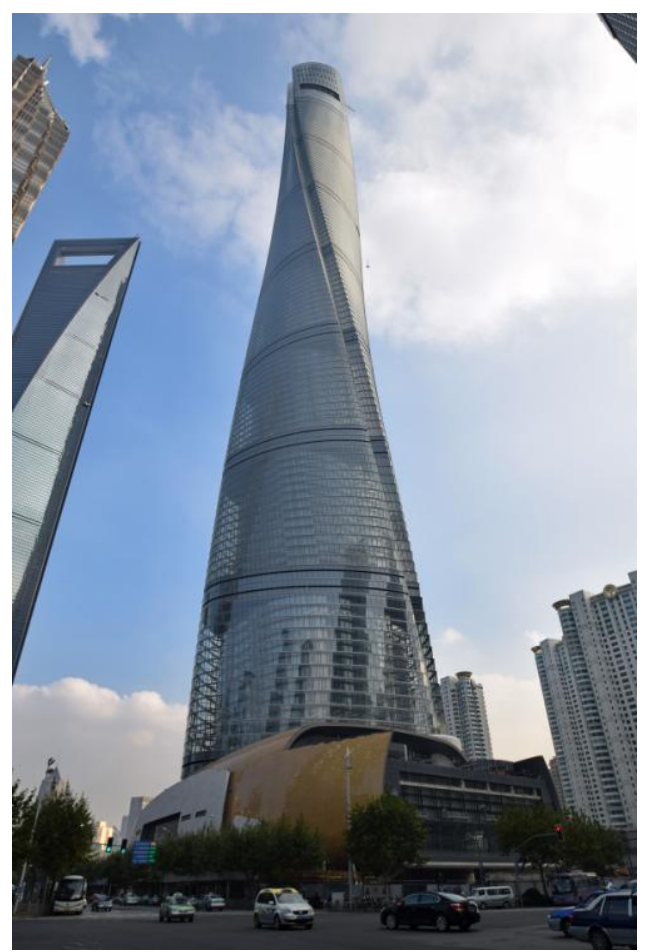

Figure 1. Shanghai Tower with double skin facades (Source: Wikipedia).

\section{DOUBLE SKIN FAÇADE DAMPING SYSTEMS}

Vortex-shedding induced across-wind vibrations are typically one of the most critical issues in structural design of tall buildings. A tall building with a DSF system is modeled as a two-degrees-of-freedom system subjected to sinusoidal loads which represent the lock-in condition due to vortex shedding. The system is composed of the primary mass (M), which corresponds to the primary building structure including the inner skin of the DSF system, and the outer skin of the DSF system $\left(\mathrm{M}_{\mathrm{d} 2}\right)$. The inner and outer skins are connected through low axial stiffness connectors $\left(\mathrm{K}_{\mathrm{d} 2}\right)$ with a damping mechanism $\left(\mathrm{C}_{\mathrm{d} 2}\right)$. The load is applied to the DSF outer skin $\left(\mathrm{M}_{\mathrm{d} 2}\right)$. Equations 1 and 2 express the dynamic amplification factors of the primary structure $(\mathrm{H})$ and that of the DSF outer skin mass $\left(\mathrm{H}_{\mathrm{d} 2}\right)$, respectively. In order to anticipate the performance of the DSF damping system shown in Figure 2, $\mathrm{H}$ and $\mathrm{H}_{\mathrm{d} 2}$ values are plotted with $\rho$ (forcing frequency to natural frequency of the primary structure ratio) values ranging from 0 to 2 . This is done repeatedly with various values for important system design parameters, such as DSF outer skin mass ratio, DSF connector stiffness and DSF connector damping. The inherent damping ratio of the primary building structure is assumed to be $1 \%$ in this study.
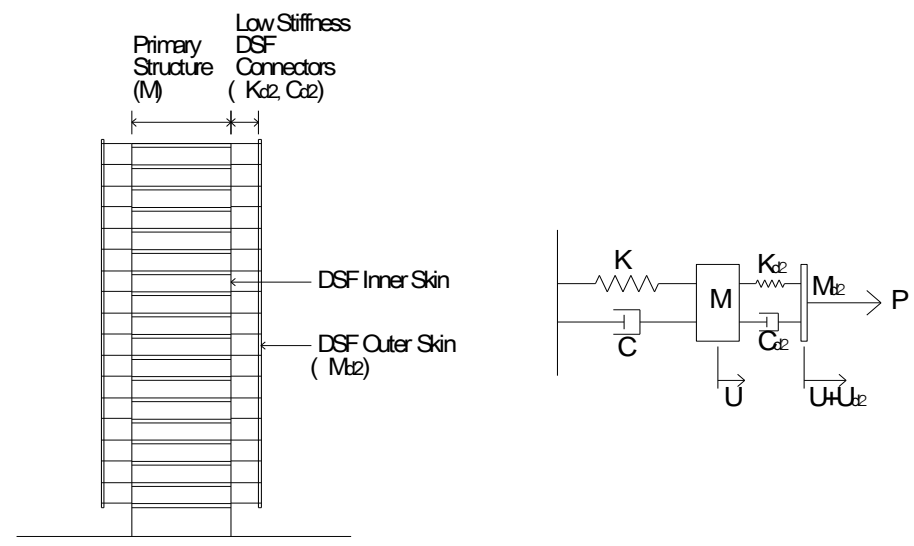

Figure 2. Simplified double skin façade damping system model. 


$$
\begin{aligned}
& H=\frac{\sqrt{f_{2}^{4}+4 f_{2}^{2} \xi_{d 2}^{2} \rho^{2}}}{\sqrt{\left(f_{2}^{2} \bar{m}_{2} \rho^{2}-\rho^{4}+\rho^{2}+f_{2}^{2} \rho^{2}-f_{2}^{2}+4 \xi \beta \xi_{k 2} f_{2}\right)^{2}+\left(2 \rho^{3} \xi_{d 2} f_{2}+2 \xi \beta-2 \xi \rho_{2}^{2}-2 f \xi_{d 2} \rho+2 m_{2} \rho^{\left.3 \xi_{d 2} f_{2}\right)^{2}}\right.}} \\
& H_{d 2}=\frac{\sqrt{\left(\rho^{2}-1\right)^{2}+4 \xi^{2} \rho^{2}}}{\bar{m}_{2} \sqrt{\left(f_{2}^{2} \bar{m}_{2} \rho^{2}-\rho^{4}+\rho^{2}+f_{2}^{2} \rho^{2}-f_{2}^{2}+4 \xi \rho \xi_{12} f_{2}\right)^{2}+\left(2 \rho^{3} \xi_{12} f_{2}+2 \xi \rho^{3}-2 \xi \phi_{2}^{2}-2 f \xi_{12} \rho+2 m_{2} \rho^{3} \xi_{12} f_{2}\right)^{2}}}
\end{aligned}
$$

$\xi=$ primary structure damping ratio

$\xi_{d 2}=\mathrm{DSF}$ connector damping ratio

$\omega=$ natural frequency of the primary structure

$\omega_{d 2}=$ natural frequency of the DSF outer skin

$\Omega=$ forcing frequency

$\bar{m}_{2}=m_{d 2} / m$

$f_{2}=\omega_{d 2} / \omega$

$\rho=\Omega / \omega$

Based on the studies with different DSF outer skin mass ratios ranging from $0.1 \%$ to $1 \%$, it was found that the DSF outer skin mass ratio is not much related to the maximum $\mathrm{H}$ value. Lowering axial stiffness of the DSF connectors reduces the maximum $\mathrm{H}$ value. The DSF outer skin to primary structure frequency ratio, $\mathrm{f}_{2}$, should be smaller than about 0.75 for the system to perform as an effective damping mechanism. However, lowering axial stiffness of the DSF connecters increases the maximum $\mathrm{H}_{\mathrm{d} 2}$ value. When the frequency ratio is close to 1 , the DSF system in Figure 2 may even perform as a force amplifier. When the frequency ratio is greater than about 1.5 , damping provided by the system is negligible.
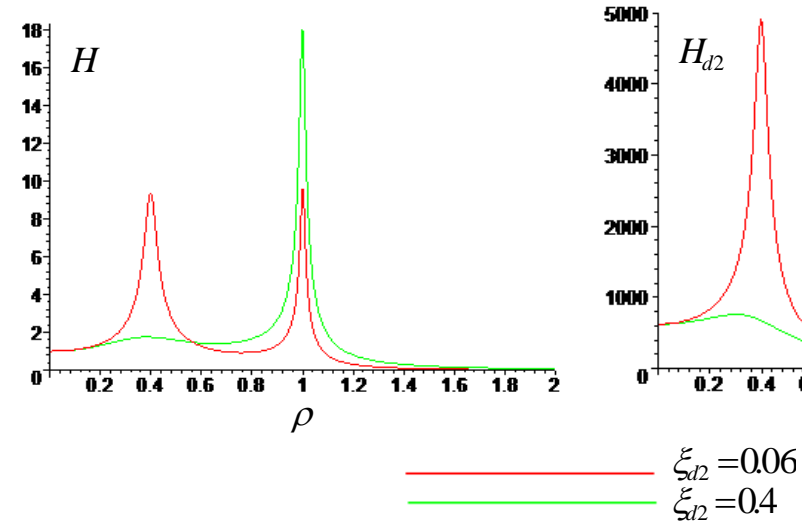

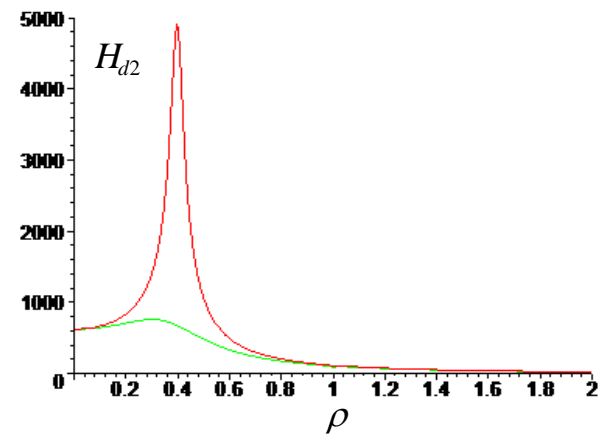

$\xi$

Figure 3. $\mathrm{H}$ and $\mathrm{H}_{\mathrm{d} 2}$ Plots of DSF damping system with $\mathrm{m}_{\mathrm{d} 2} / \mathrm{m}=0.01, \mathrm{f}_{2}=0.4, \xi=0.01, \xi_{\mathrm{d} 2}=0.06$ \& 0.4 .

Figure 3 shows example $\mathrm{H}$ and $\mathrm{H}_{\mathrm{d} 2}$ plots of the DSF damping system with the DSF outer skin to primary structure frequency ratio, $\mathrm{f}_{2}$, of 0.4 and two different connector damping ratios of 0.06 and 0.4 . The system is optimally tuned for the primary structure with the connector damping ratio of 0.06 . In this case, the maximum $\mathrm{H}$ value is about 9.5 . With the primary structure's inherent damping ratio of $1 \%$, the maximum $\mathrm{H}$ value without any auxiliary damping system is about 50. By employing the DSF damping system, the equivalent damping ratio is increased to about 5.3\%. However, with the connector damping ratio of 0.06 , the maximum $\mathrm{H}_{\mathrm{d} 2}$ value is very high. A larger DSF connector damping ratio is required to reduce the vibration of the DSF outer skins. Another plot in Figure 3 is made with the connector damping ratio of 0.4 . Now the maximum $\mathrm{H}_{\mathrm{d} 2}$ value is reduced to about $15 \%$ of the previous case. Since this is not the optimal condition for the primary structure, however, the maximum $\mathrm{H}$ value is increased to about 18 , compared to 9.5 in the previous case with the connector damping ratio of 0.06 .

As the frequency ratio is further reduced, the effectiveness of the system, in terms of mitigating the vibration of the primary structure, can be further increased. The performance of the DSF damping system with low axial stiffness 
connectors is primarily dependent on the DSF outer skin to primary structure frequency ratio. However, significantly reducing the frequency ratio may result in excessive movements of the DSF outer skin masses.

\section{VERTICALLY DISTRIBUTED TMDS WITHIN DSF CAVITIES}

The second scheme is investigated to overcome the design limitation of the first scheme, which involves movements of the DSF outer skin masses. In the second scheme, while the DSF outer skins are fixed as in the conventional DSF system, additional small masses are inserted into the DSF cavity and designed to perform as distributed tuned mass dampers (TMDs), which effectively control lateral vibrations of tall buildings under wind loads. Even though the critical design limitation of the first scheme is eliminated, the disadvantage of this scheme is that this design requires additional masses, which were not necessary in the first scheme. However, compared with the conventional TMD system, which is usually located near the top of the building, this scheme has the benefit of saving the most valuable occupiable space.

Figure 4 shows a simplified structural model representing the vertically distributed TMD system within the DSF cavities. Since TMDs installed on the floors close to the ground is ineffective, TMDs are omitted on those floors. The vertically distributed TMD system equations can be set up approximately by reducing the MDOF system primary structure to an SDOF system, considering the importance of the first mode response [3] [4]. By solving the equations, $\mathrm{H}$ (dynamic amplification factor of the primary structure) and $\mathrm{H}_{\mathrm{d}}$ (dynamic amplification factor of the TMD mass) plots can be obtained. In addition, dynamic amplification factor regarding acceleration can also be produced.

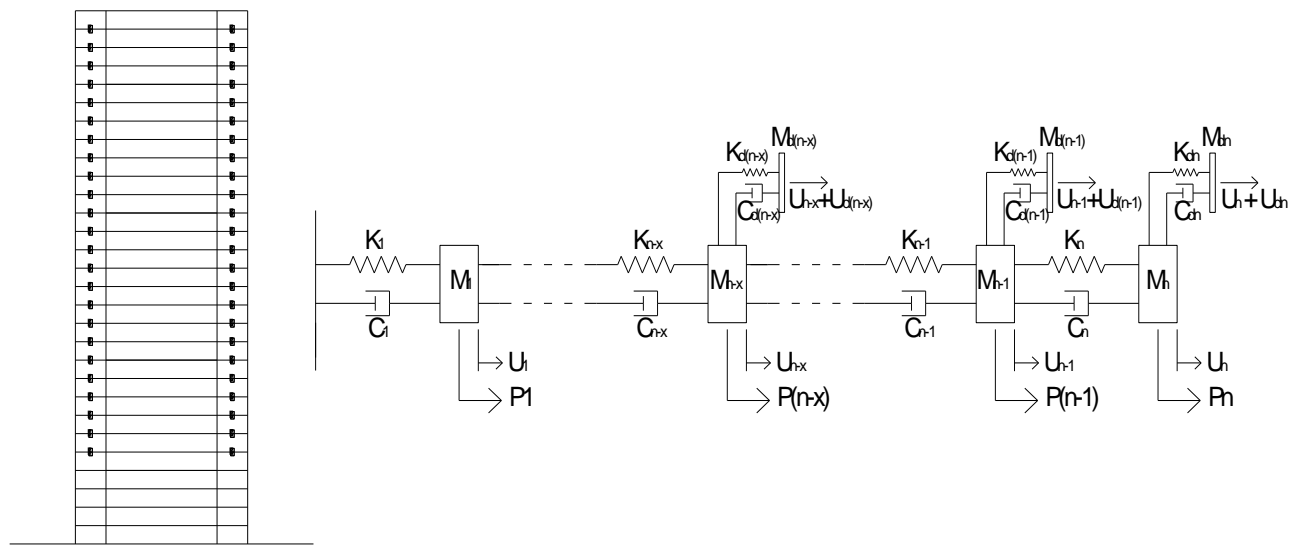

Figure 4. Simplified structural model of vertically distributed TMDs within DSF cavities.

$$
\begin{aligned}
& m \ddot{\boldsymbol{u}}+c \dot{u}+k u=p+\frac{\phi_{(n-x)}}{\phi_{n}}\left(k_{d(n-x)} u_{d(n-x)}+c_{d(n-x)} \dot{u}_{d(n-x)}\right)+\ldots .+\frac{\phi_{(n-1)}}{\phi_{n}}\left(k_{d(n-1)} u_{d(n-1)}+c_{d(n-1)} \dot{u}_{d(n-1)}\right)+\left(k_{d l} u_{d n}+c_{d n} \dot{u}_{d n}\right) \\
& m_{d(n-x)} \ddot{u}_{d(n-x)}+c_{d(n-x)} \dot{u}_{(n-x)}+k_{d(n-x)} u_{d(n-x)}=-\frac{\phi_{(n-x)}}{\phi_{n}} m_{l(n-x)} \ddot{u} \\
& m_{d(n-1)} \ddot{u}_{d(n-1)}+c_{d(n-1)} \dot{u}_{d(n-1)}+k_{d(n-1)} u_{d(n-1)}=-\frac{\phi_{(n-1)}}{\phi_{1 n}} m_{d(n-1)} \ddot{u}
\end{aligned}
$$

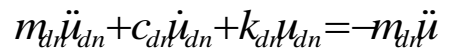

As today's tall buildings become ever taller and more slender, not only the first mode but also higher mode excitations are important design issues. In order to expand the study to investigate the higher mode responses of the system, the matrix formulation can be set up and solved using Matlab without reducing the primary structure to an SDOF system.

$$
\left.\left[\begin{array}{cc}
M & \\
& M_{d}
\end{array}\right\}\left\{\begin{array}{l}
\ddot{U} \\
\ddot{U}
\end{array}\right\}+\left[\begin{array}{cc}
C+C_{d} & -C_{d} \\
-C_{d} & C_{d}
\end{array}\right\}\left\{\begin{array}{l}
\dot{U} \\
\dot{U}_{d}
\end{array}\right\}+\left\{\begin{array}{cc}
K+K_{d} & -K_{d} \\
-K_{d} & K_{d}
\end{array}\right\} \mid \begin{array}{l}
U \\
U_{d}
\end{array}\right\}=\left\{\begin{array}{l}
P \\
0
\end{array}\right\}
$$




\section{A. Design Study I: Conventional TMD}

A 60-story building is designed as a 60-degrees-of-freedom system. Its plan dimension is 40m $\mathrm{x} 40 \mathrm{~m}$, story height is $4 \mathrm{~m}$ typical, and mass density is assumed to be $190 \mathrm{~kg} /$ cubic meter. The building is assumed to be in New York and the document SEI/ASCE 7 (Minimum Design Loads for Buildings and Other Structures) is used to establish the wind load. An inherent first mode damping ratio of $0.5 \%$ is assumed in this design study. The maximum displacement and acceleration design parameters are set to building-height/500 and 2\% of gravitational acceleration, respectively [5]. With the assumed relatively low inherent damping ratio, TMDs are required not only for the first mode but also for the second mode [6]. An equivalent damping ratio of $2.6 \%$ and $6.3 \%$ are required for the first mode displacement and acceleration controls, respectively. An equivalent damping ratio of $0.2 \%$ and $3.2 \%$ are required for the second mode displacement and acceleration controls, respectively.

In the conventional TMD design, a large single TMD is typically located near the top of the structure. In order to achieve the desired first mode equivalent damping ratio of $6.3 \%$ to meet the both displacement and acceleration design criteria, about $2.5 \%$ mass ratio is required. If the building's second mode is primarily excited in this case, the structure does not meet the acceleration criteria. In order to achieve the desired second mode equivalent damping ratio of $3.2 \%$, about $0.5 \%$ mass ratio is required. The best TMD location is where maximum displacement occurs. Thus, for a 60-story building subjected to periodic forcing, the best TMD location is at the top of the building for both the first and second mode excitations.

\section{B. Design Study II: Vertically Distributed TMDs}

When the vertical distribution of TMDs within the DSF cavities is considered in order to save the valuable occupiable space at the top of the building, smaller TMDs can be distributed according to the mode shapes. An example distribution is shown in Figure 5.

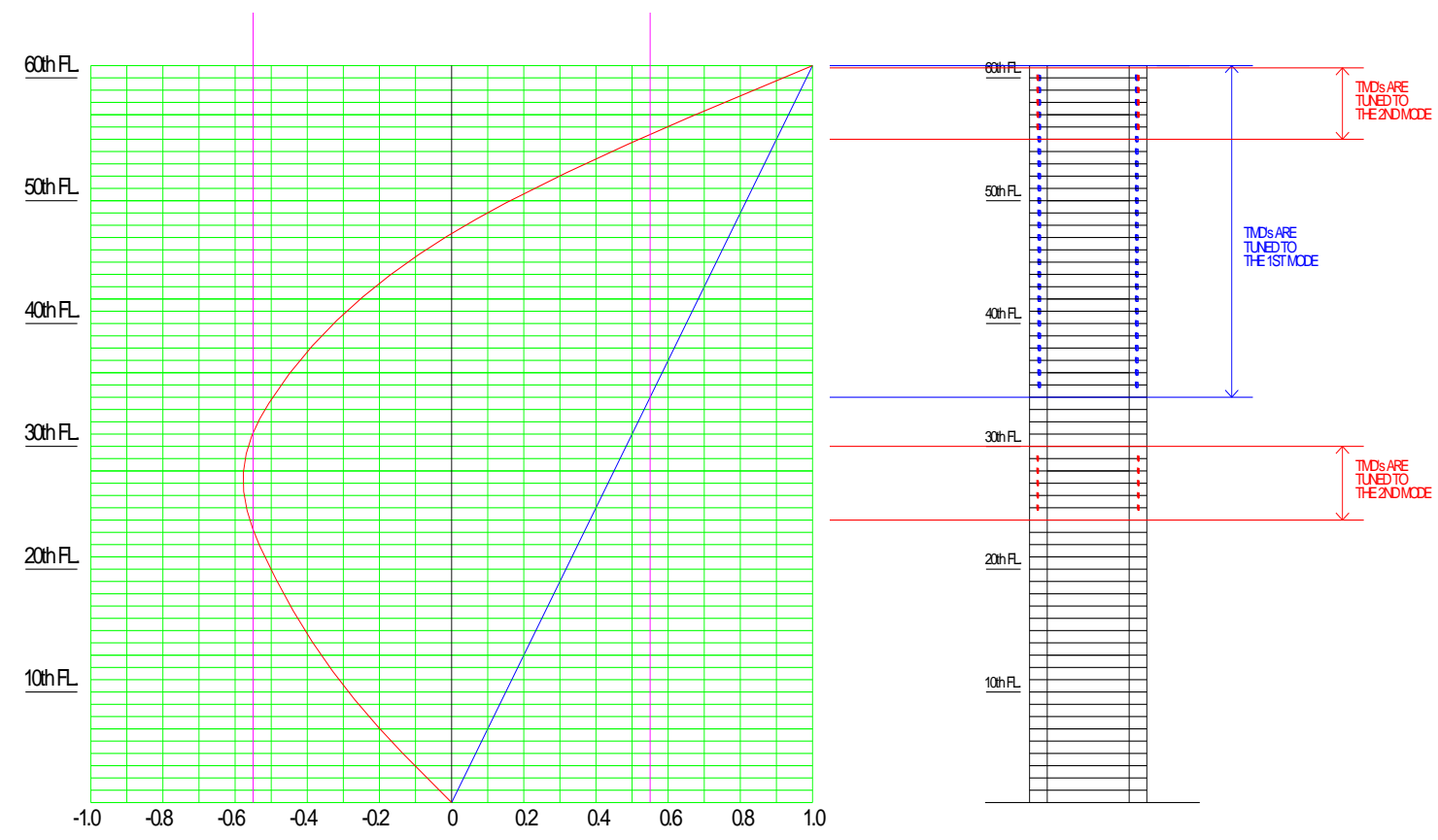

Figure 5. 60-story building with vertically distributed TMDs within DSF cavities.

Considering the desirable size and number of each small TMD, vertical distribution ranges for each mode are determined. For the first mode, TMDs are distributed from the top down to the determined floor. For the second mode control, since the mode shape is nonlinear, the vertical distribution may be divided into two zones, near the top and middle of the structure as shown in Figure 5 as an example.

In this design study, for the first mode vibration control, TMDs are distributed from node 60 to node 31 . In order to achieve the same target equivalent damping ratio, this configuration requires $67 \%$ more mass compared with the conventional system that has a single TMD at node 60. For the second mode vibration control, TMDs are distributed from node 30 to node 21 . This configuration is not as efficient as that shown in Figure 5. However, this configuration 
prevents TMD congestion near the top floors. Table 1 and Table 2 show optimal TMD tuning properties at every 5 node for the first and second mode, respectively. $M_{d}$ at each node represents the sum of possibly several dozen small TMDs.

Table -1 Vertically distributed TMDs optimal tuning properties for the $1^{\text {st }}$ mode.

\begin{tabular}{|c|c|c|c|c|c|}
\hline Node & $\mathrm{M}_{\mathrm{d}}(\mathrm{Kg})$ & $\mathrm{K}_{\mathrm{d}}(\mathrm{N} / \mathrm{m})$ & $\mathrm{f}$ & $\mathrm{Cd}(\mathrm{N}-\mathrm{s} / \mathrm{m})$ & $\varphi_{\mathrm{d}}$ \\
\hline 60 & 32800 & 30862 & 0.9700 & 7318 & 0.115 \\
\hline 55 & 32800 & 31021 & 0.9725 & 7257 & 0.114 \\
\hline 50 & 32800 & 31181 & 0.9750 & 7196 & 0.113 \\
\hline 45 & 32800 & 31341 & 0.9775 & 7134 & 0.111 \\
\hline 40 & 32800 & 31501 & 0.9800 & 7072 & 0.110 \\
\hline 35 & 32800 & 31662 & 0.9825 & 7009 & 0.109 \\
\hline 31 & 32800 & 31791 & 0.9845 & 6959 & 0.108 \\
\hline
\end{tabular}

Table -2 Vertically distributed TMDs optimal tuning properties for the $2^{\text {nd }}$ mode.

\begin{tabular}{|c|c|c|c|c|c|}
\hline Node & $\mathrm{M}_{\mathrm{d}}(\mathrm{Kg})$ & $\mathrm{K}_{\mathrm{d}}(\mathrm{N} / \mathrm{m})$ & $\mathrm{f}$ & $\mathrm{Cd}(\mathrm{N}-\mathrm{s} / \mathrm{m})$ & $\varphi_{\mathrm{d}}$ \\
\hline 30 & 25300 & 153417 & 0.9850 & 6958 & 0.055 \\
\hline 25 & 25300 & 153417 & 0.9850 & 6958 & 0.055 \\
\hline 21 & 25300 & 153417 & 0.9850 & 6958 & 0.055 \\
\hline
\end{tabular}

The motion of the TMD mass acts as a critical limiting factor in this design because the multiple small TMDs are installed within the limited DSF cavity space, the depth of which typically ranges from 0.3 to 1.5 meters [1]. When the TMDs are designed to move in the direction perpendicular to the façade plane, the motion of TMDs should be accommodated within the depth of the DSF cavity, or the DSF cavity must be wide enough to allow TMD motion for optimal performance.

One strategy to reduce TMD motion is to increase TMD damping ratio over optimal value. When the first mode of the primary structure is excited, the motions of the TMDs tuned to the first mode range from $+/-0.4 \mathrm{~m}$ to $+/-0.75 \mathrm{~m}$, and the TMD damping ratios from $10.8 \%$ to $11.5 \%$ for optimal performance. If the motions of the TMDs should be limited to maximum $+/-0.5 \mathrm{~m}$ instead due to the limitation of the DSF cavity space, this limited motion can be achieved by increasing the TMD damping ratios ranging from $15.5 \%$ to $29.5 \%$. Table 3 shows revised TMD tuning properties for this purpose for the first mode. In this case, however, the system does not produce optimal performance. The maximum acceleration is increased to about $2.8 \%$ of the gravitational acceleration, which is greater than the generally accepted maximum target value of $2 \%$. Thus, in order to resolve this acceleration problem caused by the method used to meet the TMD motion constraint, another strategy must be considered. For example, increasing TMD mass will resolve this problem, if it is acceptable. Design decisions should be made in an integrative way considering various complex and sometimes conflicting requirements.

Table -3 Vertically distributed TMDs tuning properties for the $1^{\text {st }}$ mode to meet the TMD motion constraint

\begin{tabular}{|c|c|c|c|c|c|}
\hline Node & $\mathrm{M}_{\mathrm{d}}(\mathrm{Kg})$ & $\mathrm{K}_{\mathrm{d}}(\mathrm{N} / \mathrm{m})$ & $\mathrm{f}$ & $\mathrm{Cd}(\mathrm{N}-\mathrm{s} / \mathrm{m})$ & $\varphi_{\mathrm{d}}$ \\
\hline 60 & 32800 & 30862 & 0.9700 & 18795 & 0.295 \\
\hline 55 & 32800 & 31021 & 0.9725 & 17646 & 0.277 \\
\hline 50 & 32800 & 31181 & 0.9750 & 16088 & 0.252 \\
\hline 45 & 32800 & 31341 & 0.9775 & 14448 & 0.225 \\
\hline 40 & 32800 & 31501 & 0.9800 & 12799 & 0.199 \\
\hline 35 & 32800 & 31662 & 0.9825 & 11200 & 0.174 \\
\hline 31 & 32800 & 31791 & 0.9845 & 10000 & 0.155 \\
\hline
\end{tabular}




\section{CONCLUSIONS}

Tall building dynamic motion control strategies were investigated through design integration between the primary structure and double skin façade systems. The cavity between the inner and outer skins of the DSF system was originally introduced primarily for the purpose of enhanced environmental control between the interior and exterior. In this paper, the potential of the cavity was further explored with regard to its possible contribution to structural dynamic motion control.

In the first scheme, the DSF façade connectors are designed to have low axial stiffness. This configuration reduces the transmissibility of dynamic wind loads applied to the primary structure through the connectors, and, consequently, help mitigate wind-induced vibrations of the primary structure. However, severe vibration of the DSF outer skin masses is a serious design limitation. In order to overcome this design issue, multiple small TMDs are installed within the DSF cavities in the second scheme. Compared with the conventional TMD system, no building area is occupied by conventional huge TMDs, which results in valuable space saving near the tops of tall buildings. In addition, not only first mode but also higher mode responses can effectively be controlled based on the mode shapes. Furthermore, TMDs can be more conveniently installed due to their smaller sizes compared with the conventional large TMDs. Further research is required for the practical applications of the studied systems with more improved performances.

\section{REFERENCE}

[1] Oesterle, E., Lieb, R., Lutz, M. \& Heusler, W. Double-skin Facades, Munich: Prestel, 2001.

[2] Moon, K. Tall Building Motion Control Using Double Skin Facades, ASCE Journal of Architectural Engineering, Vol. 15-3, pp 84-90, 2009.

[3] Connor, J. Introduction to Structural Motion Control. New York: Prentice Hall, 2003.

[4] Den Hartog, J. Mechanical Vibrations. $4^{\text {th }}$ Edition. New York: McGraw-Hill, 1956.

[5] Kowalczyk, R., Sinn, R. \& Kilmister, M.B. (Eds). Structural Systems for Tall Buildings (Council on Tall Buildings and Urban Habitat Monograph). New York: McGraw-Hill, 1995.

[6] Soong, T. T. \& Dargush, G.F. Passive Energy Dissipation System in Structural Engineering. New York: Wiley, 1997. 